\title{
Risk Management and Financial Derivatives: An Overview*
}

\section{Shawkat Hammoudeh}

Department of Economics

Drexel University

\author{
Michael McAleer \\ Econometric Institute \\ Erasmus School of Economics \\ Erasmus University Rotterdam \\ and \\ Tinbergen Institute \\ The Netherlands \\ and \\ Department of Quantitative Economics \\ Complutense University of Madrid \\ and \\ Institute of Economic Research \\ Kyoto University
}

April 2012

* The authors wish to thank the referees for their timely and helpful comments and suggestions on the papers comprising the special issue. The second author wishes to acknowledge the financial support of the Australian Research Council, National Science Council, Taiwan, and the Japan Society for the Promotion of Science. 


\begin{abstract}
Risk management is crucial for optimal portfolio management. One of the fastest growing areas in empirical finance is the expansion of financial derivatives. The purpose of this special issue on "Risk Management and Financial Derivatives" is to highlight some areas in which novel econometric, financial econometric and empirical finance methods have contributed significantly to the analysis of risk management, with an emphasis on financial derivatives, specifically conditional correlations and volatility spillovers between crude oil and stock index returns, pricing exotic options using the Wang transform, the rise and fall of S\&P500 variance futures, predicting volatility using Markov switching multifractal model: evidence from S\&P100 index and equity options, the performance of commodity trading advisors: a mean-variance-ratio test approach, forecasting volatility via stock return, range, trading volume and spillover effects: the case of Brazil, estimating and simulating Weibull models of risk or price durations: an application to ACD models, valuation of double trigger catastrophe options with counterparty risk, day of the week effect on the VIX - a parsimonious representation, equity and CDS sector indices: dynamic models and risk hedging, the probability of default in collateralized credit operations, risk premia in multi-national enterprises, solving replication problems in a complete market by orthogonal series expansion, downside risk management and VaR-based optimal portfolios for precious metals, oil and stocks, and implied Sharpe ratios of portfolios with options: application to Nikkei futures and listed options.
\end{abstract}

Keywords: Risk management, Optimal portfolios, Financial derivatives, Financial econometrics, Options, Futures, Volatility, Spillovers, Hedging, Default, Risk premia, Complete markets. 


\section{Introduction}

Risk management is crucial for optimal portfolio management. One of the fastest growing areas in empirical finance is the expansion of financial derivatives. While some of key the issues underlying risk and portfolio management are reasonably well understood, many of the technical and empirical issues underlying the creation and movements in financial derivatives are less well understood.

The purpose of this special issue on "Risk Management and Financial Derivatives" is to highlight some areas in which novel econometric, financial econometric and empirical finance methods have contributed significantly to the analysis of risk management, with an emphasis on financial derivatives, specifically conditional correlations and volatility spillovers between crude oil and stock index returns (Chang, McAleer and Tansuchat, 2012), pricing exotic options using the Wang transform (Labuschagne and Offwood, 2012), the rise and fall of S\&P500 variance futures (Chang, Jimenez-Martin, McAleer and Perez-Amaral, 2012), predicting volatility using Markov switching multifractal model: evidence from S\&P100 index and equity options (Chuang, Huang and Lin, 2012), the performance of commodity trading advisors: a mean-variance-ratio test approach (Bai, Phoon, Wang and Wong, 2012), forecasting volatility via stock return, range, trading volume and spillover effects: the case of Brazil (Asai and Brugal, 2012), estimating and simulating Weibull models of risk or price durations: an application to ACD models (Allen, Ng and Peiris, 2012), valuation of double trigger catastrophe options with counterparty risk (Jiang, Yang, Liu and Wang, 2012), day of the week effect on the VIX - a parsimonious representation (Gonzalez-Perez and Guerrero, 2012), equity and CDS sector indices: dynamic models and risk hedging (Caporin, 2012), the probability of default in collateralized credit operations (Divino and Rocha, 2012), risk premia in multi-national enterprises (Lutz, 2012), solving replication problems in a complete market by orthogonal series expansion (Dong and Gao, 2012), downside risk management and VaR-based optimal portfolios for precious metals, oil and stocks (Hammoudeh, Araujo Santos and Abdullah Al-Hassan, 2012), and implied Sharpe ratios 
of portfolios with options: application to Nikkei futures and listed options (Akuzawa and Nishiyama, 2012).

It is our hope that the interesting, invaluable and innovative papers in this special issue will encourage others to undertake research in a variety of challenging areas associated with the exciting and rapidly expanding areas of risk management and financial derivatives.

\section{Overview}

In the first paper, "Conditional Correlations and Volatility Spillovers Between Crude Oil and Stock Index Returns”, Chia-Lin Chang (National Chung Hsing University, Taiwan), Michael McAleer (Erasmus University Rotterdam, The Netherlands) and Roengchai Tansuchat (Maejo University, Thailand) investigate the conditional correlations and volatility spillovers between the crude oil and financial markets, based on crude oil returns and stock index returns. Daily returns from 2 January 1998 to 4 November 2009 of the crude oil spot, forward and futures prices from the WTI and Brent markets, and the FTSE100, NYSE, Dow Jones and S\&P500 stock index returns, are analysed using Bollerslev’s CCC model, Ling and McAleer's VARMA-GARCH model, McAleer, Hoti and Chan's VARMA-AGARCH model, and Engle’s DCC model. Based on the CCC model, the estimates of conditional correlations for returns across markets are very low, and some are not statistically significant, which means the conditional shocks are correlated only in the same market and not across markets. However, the DCC estimates of the conditional correlations are always significant. This result makes it clear that the assumption of constant conditional correlations is not supported empirically. Surprisingly, the empirical results from the VARMA-GARCH and VARMA-AGARCH models provide little evidence of volatility spillovers between the crude oil and financial markets. The evidence of asymmetric effects of negative and positive shocks of equal magnitude on the conditional variances suggests that VARMA-AGARCH is superior to VARMA-GARCH and CCC. The estimation and analysis of the volatility and conditional

correlations between crude oil returns and stock index returns can provide useful 
information for investors, oil traders and government agencies that are concerned with the crude oil and stock markets, especially regarding optimal hedging across the two markets.

As the second paper, Coenraad Labuschagne (University of the Witwatersrand, South Africa) and Theresa Offwood (University of the Witwatersrand, South Africa) analyse the theoretical and practical issue of "Pricing Exotic Options Using the Wang Transform”. The Wang transform allows for a simple, yet intuitive approach to pricing options with underlying based on geometric Brownian motion. The authors show how the approach by Hamada and Sherris can be used to price some exotic options. Examples are given to show the convergence of the Wang price to the Black-Scholes price for a Margrabe option, a geometric basket option and an asset-or-nothing option. The authors also examine the range of prices that is achievable using the Wang transform for these options.

Chia-Lin Chang (National Chung Hsing University, Taiwan), Juan-Angel JimenezMartin (Complutense University of Madrid, Spain), Michael McAleer (Erasmus University Rotterdam, The Netherlands) and Teodosio Perez-Amaral (Complutense University of Madrid, Spain) analyse “The Rise and Fall of S\&P500 Variance Futures” in the third paper. Modelling, monitoring and forecasting volatility are indispensible to sensible portfolio risk management. The volatility of an asset of composite index can be traded by using volatility derivatives, such as volatility and variance swaps, options and futures. The most popular volatility index is VIX, which is a key measure of market expectations of volatility, and hence also an important barometer of investor sentiment and market volatility. Investors interpret the VIX cash index as a "fear" index, and of VIX options and VIX futures as derivatives of the "fear" index. VIX is based on S\&P500 call and put options over a wide range of strike prices, and hence is not model based. Speculators can trade on volatility risk with VIX derivatives, with views on whether volatility will increase or decrease in the future, while hedgers can use volatility derivatives to avoid exposure to volatility risk. VIX and its options and futures derivatives has been widely analysed in recent years. An alternative volatility 
derivative to VIX is the S\&P500 variance futures, which is an expectation of the variance of the S\&P500 cash index. Variance futures are futures contracts written on realized variance, or standardized variance swaps. The S\&P500 variance futures are not model based, so the assumptions underlying the index do not seem to have been clearly understood. As variance futures are typically thinly traded, their returns and volatility are not easy to model accurately using a variety of volatility model specifications. The paper analyses the volatility in S\&P500 3-month and 12-month variance futures before, during and after the GFC, as well as for the full data period, for each of three alternative conditional volatility models and three densities, in order to determine whether exposure to risk can be incorporated into a financial portfolio without taking positions on the S\&P500 index itself.

In the fourth paper, entitled "Predicting Volatility Using Markov Switching Multifractal Model: Evidence from S\&P 100 Index and Equity Options”, Wen-I Chuang (National Taiwan University, Taiwan), Teng-Ching Huang (National Taiwan University of Sciences and Technology, Taiwan) and Bing-Huei Lin (National Chung Hsing University, Taiwan) evaluate the performance of the ability of Markov-switching multifractal (MSM), implied, GARCH, and historical volatilities to predict realized volatility for both the S\&P100 index and equity options. Some important findings are as follows. First, the authors find that the ability of MSM and GARCH volatilities to predict realized volatility is better than that of implied and historical volatilities for both the index and equity options. Second, equity option volatility is more difficult to be forecast than index option volatility. Third, both index and equity option volatilities can be better forecast during non-global financial crisis periods than during global financial crisis periods. Fourth, equity option volatility exhibits distinct patterns conditional on various equity and option characteristics and its predictability by MSM and implied volatilities depends on these characteristics. Finally, the authors find that MSM volatility outperforms implied volatility in predicting equity option volatility conditional on various equity and option characteristics.

Zhidong Bai (North East Normal University, China), Kok Fai Phoon (Singapore 
Management University, Singapore), Keyan Wang (Shanghai Finance University, China) and Wing-Keung Wong (Hong Kong Baptist University, China) examine "The Performance of Commodity Trading Advisors: A Mean-Variance-Ratio Test Approach” in the fifth paper. The authors provide simulation evidence that the mean-variance-ratio (MVR) test is superior to the Sharpe-ratio (SR) test by applying both tests to analyze the performance of commodity trading advisors (CTAs). The empirical findings show that while the SR test concludes that most of the CTA funds being analyzed are indistinguishable in their performance, the MVR statistic shows that some funds outperformed others. Moreover, the SR statistic indicates that one fund significantly outperformed another, even when the difference between the two funds was insignificant or even changed directions over sub-periods. Conversely, the MVR statistic can detect such changes when they occur in the sub-periods. In addition, the authors have conducted simulations to show that the MVR test possesses good power, which should be very helpful in practical applications.

In the sixth paper, entitled "Forecasting Volatility via Stock Return, Range, Trading Volume and Spillover Effects: The Case of Brazil”, for the purpose of developing alternative approach for forecasting volatility, Manabu Asai (Soka University, Japan) and Ivan Brugal (Soka University, Japan and Superintendent of Banks, Dominican Republic) consider the Heterogeneous VAR (HVAR) model which accommodates the market effects of different horizons, namely daily, weekly and monthly effects. The authors examine the interdependence of stock markets in Brazil and the USA, based on information of daily return, range and trading volume. For purposes of comparison with the new approach, the authors also analyse the univariate and multivariate GARCH models with asymmetric effects, trading volumes and fat-tails. The heteroskedasticitycorrected Granger causality tests based on the HVAR model show strong evidence of such spillover effects. The authors assess the Value-at-Risk thresholds for Brazil, based on the out-of-sample forecasts of the HVAR model, and find that the new approach is satisfactory for periods that include the GFC, without assuming heavy-tailed conditional distributions. 
David Allen (Edith Cowan University, Australia), K.H. Ng (University of Malaya, Malaysia) and Shelton Peiris (University of Sydney, Australia) consider "Estimating and Simulating Weibull Models of Risk or Price Durations: An Application to ACD Models” in the seventh paper. There is now a massive literature on both the GARCH family of risk models and the related Auto-Conditional Duration (ACD) Models used for modelling the stochastic timing of trades or price changes in finance market microstructure research. Both have their origins in the classic (G)ARCH papers by Engle and Bollerslev. The paper uses the theory of estimating functions (EF) as a semi-parametric method for estimating the parameters of this type of model. As an example, the authors consider the class of ACD models with errors from the standard Weibull distribution to develop an estimation procedure. This method could equally be applied to GARCH models. Using a large scale simulation study, the authors show that the EF approach is easier to use in practice than the maximum likelihood (ML) or quasi-maximum likelihood (QML) methods. The statistical properties of the corresponding optimal estimates are investigated, and it is shown that the estimates using both the EF and QML methods are comparable. However, the EF estimates are easier to evaluate than the ML and QML methods. Nevertheless, ML-based estimates are are shown to be superior and perform better when the true distribution is known but, when this is not the case, the EF estimates are shown to be a powerful tool.

In the eighth paper, entitled "Valuation of Double Trigger Catastrophe Options With Counterparty Risk”, I-Ming Jiang (Yuan Ze University, Taiwan), Sheng-Yung Yang (National Chung Hsing University, Taiwan), Yu-Hong Liu (National Cheng Kung University, Taiwan) and Alan T. Wang (National Cheng Kung University, Taiwan) present a novel catastrophe option pricing model that considers counterparty risk. Asset prices are modelled through a jump-diffusion process which is correlated with counterparty loss process and collateral assets. Owing to the long term of catastrophe options, the authors also examine the model in the interesting stochastic interest rate environment. The numerical results indicate that counterparty risk significantly affects the value of options. Recently, numerous serious financial events have demonstrated the 
importance of counterparty risk when valuing financial products, so the results of the paper should be of practical importance.

Maria-Teresa Gonzalez-Perez (CUNEF, Madrid, Spain) and David E. Guerrero (CUNEF, Madrid, Spain) consider the "Day of the Week Effect on the VIX. A Parsimonious Representation" in the ninth paper. The paper examines significant deterministic seasonal patterns in financial asset returns to be of great importance to academics and investors. The authors analyze the presence of seasonal daily patterns in the VIX and S\&P 500 returns series using a trigonometric specification. First, the authors show that, given the isomorphism between the trigonometrical and alternative seasonality representations (that is, daily dummies), it is possible to test daily seasonal patterns by employing a trigonometrical representation based on a finite sum of weighted sines and cosines. The authors find a potential evolutionary seasonal pattern in the daily VIX that is not in the daily S\&P 500 log-returns series. In particular, they find an inverted Monday effect in the VIX level and changes in the VIX, and a U-shaped seasonal pattern in the changes in the VIX when they control for outliers. The trigonometrical representation is more robust to outliers than the one commonly used by literature, but it is not immune to them. Finally, the authors do not find a day-of-the-week effect in S\&P500 returns series, which suggests the presence of a deterministic seasonal pattern in the relation between VIX and S\&P 500 returns.

In the tenth paper, entitled "Equity and CDS Sector Indices: Dynamic Models and Risk Hedging”, Massimiliano Caporin (University of Padova, Italy) examines the recent GFC as having had a substantial impact on equity and bond markets, as well as on the performances of managed portfolios which have been hit by the decrease of both indices. Nevertheless, the availability of indices monitoring the equity market volatility, the VIX index, credit markets default risk, and CDS indices, allows for the construction of hedging strategies. In this paper, the author takes the point of view of an equity investor who wants to hedge equity risk by taking positions either on the VIX index or on CDS indices. In deriving the dynamic hedge ratios, the author considers the joint system dynamics of alternative variables, taking into account mean relations, variance spillovers, 
and asymmetry, as well as correlation changes over time. The analysis is based on sectoral indices and shows the advantages of hedging and the impact of a model specification.

Jose Angelo Divino (Catholic University of Brasilia, Brazil) and Líneke Clementino Sleegers Rocha (Catholic University of Brasilia and Caixa Economica Federal, Brazil) analyze the "Probability of Default in Collateralized Credit Operations" in the eleventh paper. The authors identify the major determinants of the probability of default in a mortgage credit operation, which is backed by collateral. The authors use an exclusive and novel data set with 268,036 loan contracts and apply logistic regression and Cox proportional hazards model in estimation. The discriminatory power of the estimated models is analyzed using several accuracy indicators. The inclusion of time-dependent macroeconomic variables, in addition to covariates representing characteristics of the contract and individuals, improve the overall empirical performance. Logistic regression shows higher discriminatory power than Cox proportional hazards model according to all the accuracy indicators. It is worth mentioning the negative relationship between the probability of default and the economy base interest rate. Decreases in the base interest rate lead banks to lose revenue from treasury operations and expand credit operations to compensate the loss. This strategy brings individuals with a higher probability of default to the financial market.

In the twelfth paper, Stefan Lutz (University of Manchester, UK and Complutense University of Madrid, Spain) evaluates "Risk Premia in Multi-national Enterprises”. The author commences with the well-known result that the CAPM implies that investors require equity risk premia when choosing risky investments. Therefore, investors will demand higher returns to equity invested if higher risk is present. This should also apply to investments in independent enterprises and multi-national enterprises alike. This hypothesis is investigated by analyzing an interesting and large panel data set of 407,000 European firms for the years 1985 to 2010. When income is set in relation to invested capital, risk measured by earnings volatility emerges as the most important stable determinant of income. The empirical results indicate that both MNEs and independent 
firms regularly account for risk as a major determinant of income when pricing international goods and services. Hence, it is argued that international taxation rules for multi-national enterprises should account for risk premia in transfer prices and resulting profits.

Chaohua Dong (Monash University, Australia) and Jiti Gao (Monash University, Australia) solve analytical replication problems in a complete market by orthogonal series expansion in the thirteenth paper. The authors reconsider the replication problem for contingent claims in a complete market under a general framework. As there are various limitations in the Black-Scholes pricing formula, they propose a new method to obtain a practical and explicit self-financing trading strategy expression for replications of claims in a general model. The main advantage of the new method is the use of an orthogonal expansion method to derive a closed-form expression for the self-financing strategy that is associated with some general underlying asset processes. As a consequence, a replication strategy is obtained for a European option. Converse to the traditional Black-Scholes theory, the authors derive a pricing formula for a European option from the proposed replication strategy that is quite different from the BlackScholes pricing formula. The authors provide an implementation procedure and both numerical and empirical examples to show how the proposed trading strategy works in practice, and compare the results with a replication strategy based on the Black-Scholes theory.

In the penultimate paper, Shawkat Hammoudeh (Drexel University, USA), Paulo Araujo Santos (University of Lisbon, Portugal) and Abdullah Al-Hassan (IMF, USA) analyse the topical issue of constructing and forecasting Value-at-Risk (VaR), which is used to analyze the market downside risk associated with investments in six key individual assets including four precious metals, oil and the S\&P 500 index, and three diversified portfolios. Using combinations of these assets, three optimal portfolios and their efficient frontiers within a VaR framework are constructed and the returns and downside risks for these portfolios are also analyzed. One-day-ahead VaR forecasts are computed with nine risk models including calibrated RiskMetrics, asymmetric GARCH-type models, the 
filtered Historical Simulation approach, methodologies from statistics of extremes, and a risk management strategy involving combinations of models. These risk models are evaluated and compared based on the unconditional coverage, independence and conditional coverage criteria. The economic importance of the results is also highlighted by assessing the daily capital charges under the Basel Accord rule. The best approaches for estimating the VaR for individual assets and for the three VaR-based optimal portfolios and efficient frontiers are discussed. The VaR-based performance measure ranks the most diversified optimal portfolio as the most efficient and the pure precious metals as the least efficient.

Toshinao Akuzawa (Nomura Securities and Kyoto University, Japan) and Yoshihiko Nishiyama (Kyoto University, Japan) examine implied Sharpe ratios of portfolios with options: application to Nikkei futures and listed options” in the fourteenth and final paper. The authors propose a practical criterion for portfolio selection, namely the implied excess Sharpe ratio, which is intended as an excess Sharpe ratio (versus the underlying stock) that investors can expect to enjoy from portfolios that include options. This should be a useful ex ante indicator that can be easily calculated. There are many ways to include options in a portfolio, but the combination that produces the largest implied excess Sharpe ratio is the best way to maximize the short-term Sharpe ratio. The selection process does not require historical simulation or prediction of share price average growth rates, and is highly transparent as it can be easily reproduced (at a low calculation cost). Hence, the implied excess Sharpe ratio should be an effective tool for investors seeking returns in exchange for a certain amount of risk that want to use the options market efficiently. The short-term Sharpe ratio is not necessarily the only criterion, but is a rational benchmark of portfolio performance that is closely related to criteria such as the long-term Sharpe ratio and maximum drawdown. In order to examine the benefit of the concept, the authors construct an investment strategy that automatically selects from multiple candidate portfolios that are made up of combinations of Nikkei futures and Nikkei listed options of the portfolio with the largest implied excess Sharpe ratio. Back-testing shows that this investment strategy also performs well over the long term. 


\section{Final remarks}

It is our hope that the collection of interesting, invaluable and innovative papers in this special issue by some of the leading experts in the field of "Risk Management and Financial Derivatives” will be of wide interest to theoreticians and practitioners alike in risk and portfolio management, empirical finance and financial econometrics, and will encourage others to undertake research in a variety of challenging areas associated with the exciting and rapidly expanding areas of risk management and financial derivatives. It is our pleasure to acknowledge all the contributors for preparing their interesting and innovative papers in a timely manner, and for actively participating in the rigorous review process. 


\section{References}

Akuzawa, T. and Y. Nishiyama (2012), "Implied Sharpe Ratios of Portfolios With Options: Application to Nikkei Futures and Listed Options”, to appear in North American Journal of Economics and Finance, this issue.

Allen, D., K.H. Ng and S. Peiris (2012), "Estimating and Simulating Weibull Models of Risk or Price Durations: An Application to ACD Models", to appear in North American Journal of Economics and Finance, this issue.

Asai, M. and I. Brugal (2012), "Forecasting Volatility via Stock Return, Range, Trading Volume and Spillover Effects: The Case of Brazil”, to appear in North American Journal of Economics and Finance, this issue.

Bai, Z., K.F. Phoon, K. Wang and W.-K. Wong (2012), “The Performance of Commodity Trading Advisors: A Mean-Variance-Ratio Test Approach”, to appear in North American Journal of Economics and Finance, this issue.

Caporin, M. (2012), “Equity and CDS Sector Indices: Dynamic Models and Risk Hedging”, to appear in North American Journal of Economics and Finance, this issue.

Chang, C.-L., J.-A. Jimenez-Martin, M. McAleer and T. Perez-Amaral (2012), “The Rise and Fall of S\&P500 Variance Futures”, to appear in North American Journal of Economics and Finance, this issue.

Chang, C.-L., M. McAleer and R. Tansuchat (2012), "Conditional Correlations and Volatility Spillovers Between Crude Oil and Stock Index Returns”, to appear in North American Journal of Economics and Finance, this issue.

Chuang, W.-I, T.-C. Huang and B.-H. Lin (2012), "Predicting Volatility Using Markov Switching Multifractal Model: Evidence from S\&P 100 Index and Equity Options”, to appear in North American Journal of Economics and Finance, this issue.

Divino, J.A. and L.C.S. Rocha (2012), "Probability of Default in Collateralized Credit Operations”, to appear in North American Journal of Economics and Finance, this issue.

Dong, C. and J. Gao (2012), "Solving Replication Problems in a Complete Market by Orthogonal Series Expansion”, to appear in North American Journal of Economics and Finance, this issue.

Gonzalez-Perez, M.-T. and D.E. Guerrero (2012), "Day of the Week Effect on the VIX. A Parsimonious Representation", to appear in North American Journal of Economics and Finance, this issue. 
Shawkat, S., P. Araujo Santos and A. Al-Hassan (2012), "Downside Risk Management and VaR-based Optimal Portfolios for Precious Metals, Oil and Stocks”, to appear in North American Journal of Economics and Finance, this issue.

Jiang, I-M., S.-Y. Yang, Y.-H. Liu and A.T. Wang (2012), "Valuation of Double Trigger Catastrophe Options with Counterparty Risk", to appear in North American Journal of Economics and Finance, this issue.

Labuschagne, C. and T. Offwood (2012), "Pricing Exotic Options Using the Wang Transform”, to appear in North American Journal of Economics and Finance, this issue.

Lutz, S. (2012), "Risk Premia in Multi-national Enterprises", to appear in North American Journal of Economics and Finance, this issue. 